\title{
The Efficacy of a Chinstrap in Treating Sleep Disordered Breathing and Snoring
}

Sushanth Bhat, M.D. '; Neola Gushway-Henry, M.D.'; Peter G. Polos, M.D., Ph.D.'; Vincent A. DeBari, Ph.D.2; Sandeep Riar, M.D.3; Divya Gupta, M.D., F.A.A.S.M. ${ }^{1}$; Liudmila Lysenko, M.D. ${ }^{4}$; Disha Patel, M.D. ${ }^{1}$; Justin Pi, M.D.5; Sudhansu Chokroverty, M.D., F.A.A.S.M. ${ }^{1}$

${ }^{1}$ NJ Neuroscience Institute at JFK Medical Center/Seton Hall University, Edison NJ; ${ }^{2}$ Seton Hall University School of Health and Medical Sciences, South Orange, NJ; ${ }^{3} J F K$ Medical Center, Edison, NJ; ${ }^{4}$ Ochsner Health Systems, New Orleans, LA; ${ }^{5}$ Hackensack University Medical Center, Hackensack NJ

Study Objectives: A previously published case report suggested that a chinstrap alone might improve obstructive sleep apnea (OSA). We conducted this study to determine whether a chinstrap was a feasible alternative to continuous positive airway pressure (CPAP) in patients with OSA.

Methods: 26 adult patients with OSA (apnea-hypopnea index $[\mathrm{AHI}]>5 /$ h on diagnostic polysomnogram [PSG]) underwent a modified split-night PSG, using only a chinstrap for the first 2 hours of sleep, followed by CPAP titration for the remainder of the night. Improvements in $\mathrm{AHI}$, arterial oxygen saturation $\left(\mathrm{SpO}_{2}\right)$, and snoring with chinstrap use were compared to results with optimal CPAP pressures.

Results: There was no significant difference between the diagnostic PSG and the chinstrap portion of the split-night PSG in the following parameters: general AHI (median [IQR] 16.0/h [9.7-26.0] vs. 25.9/h [10.7-42.7]), $\mathrm{SpO}_{2}$ nadir (84.0\% [80.5-87.5] vs. 87.0 [84.0-88.5]), AHI in REM sleep (26.7/h [16.8-43.7] vs. $42.4 / \mathrm{h}$ [21.3-57.7]), $\mathrm{AHI}$ in supine sleep (24.9/h [11.9-51.5] vs. 29.8/h [11.7-55.5]), snoring index (253.2/h [147.5-353.1] vs. $180.0 / \mathrm{h}$ [9.8-393.3]) or subjective snoring scale (3.0 [0.8-3.0] vs. 2.5 [0.4-3.0]). The $\mathrm{AHI}$ and $\mathrm{SpO}_{2}$ nadir in the 13 patients with mild OSA also did not improve with chinstrap use (9.6/h [8.1-12.2] vs. $10.6 / \mathrm{h}$ [6.8-35.4] and $87.0 \%$ [83.0-90.0] vs. $88.0 \%$ [87.0-89.0]). All these parameters showed significant improvement with optimal CPAP titration $(p<0.05)$.

Conclusions: A chinstrap alone is not an effective treatment for OSA. It does not improve sleep disordered breathing, even in mild OSA, nor does it improve the AHI in REM sleep or supine sleep. It is also ineffective in improving snoring.

Keywords: obstructive sleep apnea, chinstrap, alternatives to CPAP, polysomnography, modified split-night polysomnography, snoring, positional OSA, REM OSA

Citation: Bhat S, Gushway-Henry N, Polos PG, DeBari VA, Riar S, Gupta D, Lysenko L, Patel D, Pi J, Chokroverty S. The efficacy of a chinstrap in treating sleep disordered breathing and snoring. J Clin Sleep Med 2014;10(8):887-892.
$\mathrm{O}$ bstructive sleep apnea (OSA) is a disorder characterized by repeated episodes of upper airway narrowing and closure in sleep, with subsequent arterial oxygen $\left(\mathrm{SpO}_{2}\right)$ desaturations and repeated arousals from sleep. It is a common condition, reported in up to $10 \%$ of men and $3 \%$ of women aged 30-49 years; between the ages of 50-70 years the prevalence goes up to $17 \%$ and $9 \%$, respectively. ${ }^{1}$ OSA results in sleep fragmentation, excessive daytime sleepiness, and cognitive complaints, and is a major cause of motor vehicle accidents. ${ }^{2}$ In addition, it carries significant cardiovascular morbidity due to the cumulative burden of recurrent hypoxia and sympathetic hyperactivity. ${ }^{3}$ The most uniformly effective and first-line treatment, continuous positive airway pressure (CPAP), is, unfortunately, often poorly tolerated. ${ }^{4-6}$ For this reason, efforts to find effective and more acceptable alternatives to CPAP treatment are continuing; currently available options include dental appliances, ${ }^{7}$ negative pressure oral devices, ${ }^{8}$ nasal end-expiratory devices, ${ }^{9}$ and various orthodontic, maxillofacial and palatal surgical procedures. ${ }^{10,11}$ Most of these alternative therapies are, however, effective only in selected subsets of patients with OSA.

Vorona and colleagues ${ }^{12}$ published an interesting case report in which a patient with severe OSA (apnea-hypopnea index [AHI] 42/h in general, 44/h in REM sleep as detected by overnight split-night polysomnography [PSG]) discontinued CPAP

\begin{abstract}
BRIEF SUMMARY
Current Knowledge/Study Rationale: This study addresses questions raised by a previously published case report that suggests that a chinstrap alone may be an alternative to CPAP in the treatment of OSA. We systematically analyzed the effects of chinstrap use on sleep disordered breathing based on severity of OSA in REM sleep and in supine sleep, and also addressed the question of whether the use of a chinstrap alone improves snoring.

Study Impact: The study results suggest that a chinstrap alone is an ineffective treatment for sleep disordered breathing, even in patients with mild OSA, and does not improve snoring. It also does not improve the AHI in REM sleep or supine sleep.
\end{abstract}

therapy after one month and wore a chinstrap alone to treat his OSA, with continued subjective clinical benefit. A repeat PSG was performed three months after the patient's initial study (at which point he had not been using CPAP for two months), with the patient wearing a chinstrap without CPAP, and it showed that his AHI had normalized to $1 / \mathrm{h}$. Separate AHIs in REM sleep and supine sleep were not reported, but it was not felt that differences in sleep stage or position between the two studies explained the elimination of OSA by chinstrap use. Finding the use of a chinstrap alone more efficacious than CPAP therapy in treating OSA in this particular patient, Vorona 
and colleagues recommended that the use of a chinstrap alone be investigated as a possible alternative to CPAP in the treatment of OSA. Subsequent to the publication of this case report, advertisements and discussions began appearing on the internet and other public media marketing chinstraps alone as treatment options for OSA and snoring.

We therefore conducted this prospective study to determine if a chinstrap alone was indeed a feasible alternative to CPAP in the treatment of OSA, whether a particular subset of patients (based on the severity of OSA as determined by the AHI) would benefit more than others, and whether it would improve the AHI in REM sleep or in supine sleep. We also attempted to determine if snoring, as an independent symptom, would improve with the use of a chinstrap.

\section{METHODS}

Twenty-six adult patients (ages ranging from 22 to 57 years old) who were seen in the sleep clinic at the NJ Neuroscience Institute at JFK Medical Center in Edison, NJ, between August 2011 and September 2012, and who had had PSGs that showed an $\mathrm{AHI} \geq 5 / \mathrm{h}$ were enrolled in the study. Written informed consent was obtained and the study was approved by the Institutional Review Board at JFK Medical Center, Edison, NJ. In addition to studying all patients as a single group, we classified them into 2 subgroups based on their AHIs; those with an $\mathrm{AHI} \leq 14.9 / \mathrm{h}$ were classified as having mild OSA, and those with an AHI $\geq 15 / \mathrm{h}$ were classified as having moderate-tosevere OSA. After enrollment, all patients underwent a modified split-night study as follows; per protocol, the technician was instructed to perform a PSG for 2 initial hours of sleep with the patient using a chinstrap (ResMed Chin Restraint brand) alone (without CPAP), followed by CPAP titration per established protocol for the rest of the night to eliminate sleep disordered breathing (SDB). However, the ranges of total sleep time (TST) with patients wearing the chinstrap alone ranged from $81 \mathrm{~min}$ to $179.5 \mathrm{~min}$, excluding a single patient who had both a full-night PSG wearing a chinstrap alone (with a TST $360.5 \mathrm{~min}$ ) and then a full-night CPAP titration study based on his stated preference. All PSGs were performed using hardware (Comet-PLUS XL lab-based PSG) and software (TWin PSG Clinical Software) developed by GRASS Technology (Natus Neurology, Inc, Warwick, RI, USA). Standard measurements included 4 to 8 channels for electroencephalography, as well as channels for electrooculography, submental and bilateral tibial electromyography and electrocardiography, nasal airflow measurement using nasal cannulae connected to a pressure transducer (SleepSense, SLP Inc., St. Charles IL), oral airflow using an oronasal thermistor (Braebon Medical Corp., Kanata, ON, Canada), effort measurement using chest and abdominal respiratory impedance plethysmography belts (SleepSense, SLP Inc., St. Charles, IL), and arterial oxygen saturation using a pulse oximeter (SleepSense, SLP Inc., St. Charles, IL). Snoring was detected using a microphone placed over the patient's neck (Pro-Tech Services, Inc., Murrysville PA), which provided a snoring index (defined as the number of snoring events $/ \mathrm{h}$ ). In addition, snoring was subjectively scored by the technician based on an ordinal scale developed in our laboratory $(0=$ none, $1=$ mild, $2=$ moderate, $3=$ severe $)$ using a wall-mounted microphone system (Louroe Electronics, Van Nuys CA).

All PSGs were reviewed by board certified sleep medicine specialists. We used standard 2007 American Academy of Sleep Medicine criteria to score sleep stages and respiratory events. Apneas were defined as events lasting $\geq 10 \mathrm{sec}$ and accompanied by $\geq 90 \%$ reduction in airflow for $\geq 90 \%$ of the event (if respiratory effort persisted, it was described as an obstructive apnea, and if effort was absent, it was described as a central apnea). A hypopnea was defined as a reduction in airflow $\geq 50 \%$ lasting $\geq 10 \mathrm{sec}$ and accompanied by $\mathrm{SpO}_{2}$ desaturation $\geq 3 \%$ or an arousal. ${ }^{13}$ Position was scored by video analysis. Based on the results of the CPAP titration component of the modified split-night study, each patient was assigned an "optimal CPAP pressure," which was the CPAP or bilevel pressure tested during the study that was considered by the interpreting polysomnographer to be most effective in eliminating SDB. In 11 studies, no CPAP or bilevel pressure tried was deemed to be adequate to treat the patient's SDB, and these patients were ultimately prescribed either a pressure greater than the highest pressure studied during the titration (5 patients), or were prescribed an autotitrating device (6 patients) as part of their long-term clinical treatment. In these cases, the highest pressure tried during the titration portion of the split-night study was designated as the "optimum CPAP titration pressure" for purposes of this research study. We then compared various respiratory parameters across the 3 "studies" - the baseline PSG study ("diagnostic PSG study"), the portion of the modified split-night study when the patient was wearing a chinstrap ("chinstrap study"), and the optimal CPAP pressure on the CPAP portion of the modified split-night study ("optimal CPAP study").

This study was powered to detect a reduction of AHI from $20 / \mathrm{h}$ to $5 / \mathrm{h}$ with a pooled standard deviation of $10 / \mathrm{h}$. At $\alpha=0.05$ and $\beta=0.05$ (power $=95 \%$ ), normally distributed data would require $\mathrm{n}=8$. To account for the likelihood of non-normally distributed data, we applied a $15 \%$ increase to account for the maximum asymptotic relative efficiency ${ }^{14}$ for a multiple groupwise comparison. This resulted in a requirement of 9 completed studies. Because this number was believed to be statistically adequate but lacking in generalizability, we increased the number to 26 .

Data were tested for normality by the D'Agostino-Pearson omnibus normality test. For the application of parametric statistics, we required all groups within a given comparison to be normally distributed, in which case, we used a repeated measures analysis of variance (ANOVA) and the Student Newman-Keuls test, post hoc. For comparisons in which one or more groups were not normally distributed, we used a nonparametric, repeated measures test (Friedman test) followed by Dunn test post hoc.

For statistical significance, we required $\mathrm{p}<\alpha$, i.e., $\mathrm{p}<0.05$ (two-tailed). All calculations were made using Prism software (GraphPad Corp., San Diego, CA, USA) on a Windows 7/ personal computer platform.

\section{RESULTS}

Patient demographics, diagnostic PSG data and optimal CPAP titration pressures are presented in Table 1. The mild and 
Table 1-Baseline characteristics, PSG characteristics, and optimal CPAP pressure settings of patients in the study

\begin{tabular}{|c|c|c|c|}
\hline & $\begin{array}{l}\text { All patients } \\
(\mathrm{N}=26)\end{array}$ & $\begin{array}{l}\text { Mild OSA group } \\
\quad(n=12)\end{array}$ & $\begin{array}{l}\text { Moderate-to-severe OSA group } \\
\qquad(\mathrm{n}=14)\end{array}$ \\
\hline Age (years) & $48(41-55)$ & $43(40-52)$ & $54(43-58)$ \\
\hline Gender & $\begin{array}{r}\text { Males } 15(57 \%) \\
\text { Females } 11(43 \%)\end{array}$ & $\begin{array}{r}\text { Males } 6(50 \%) \\
\text { Females } 6(50 \%)\end{array}$ & $\begin{array}{r}\text { Males } 9(64 \%) \\
\text { Females } 5(36 \%)\end{array}$ \\
\hline $\mathrm{BMI}\left(\mathrm{kg} / \mathrm{m}^{2}\right)$ & $31(27-38)$ & $36(27-40)$ & $31(27-35)$ \\
\hline Mallampati score & $4(3-4)$ & $3(3-4)$ & $4(3-4)$ \\
\hline Neck circumference (inches) & $16.5(15.5-17.0)$ & $16.5(15.5-17.0)$ & $17.0(15.6-17.4)$ \\
\hline $\begin{array}{l}\text { Percentage of TST with } \mathrm{SpO}_{2} \text { below } \\
90 \% \text { on diagnostic PSG }\end{array}$ & $2.1(0.4-4.0)$ & $0.3(0.0-3.3)$ & $2.6(1.0-10.1)$ \\
\hline $\begin{array}{l}\text { Optimum CPAP / bilevel pressure } \\
\text { (IPAP / EPAP) in } \mathrm{cm}_{2} \mathrm{O}\end{array}$ & $10(7-15) / 10(7-14)$ & $9(7-14) / 9(7-14)$ & $11(7-14)$ \\
\hline
\end{tabular}

All values expressed as median, interquartile range. CPAP, continuous positive airway pressure. EPAP, expiratory positive airway pressure. IPAP, inspiratory positive airway pressure. PSG, polysomnogram. OSA, obstructive sleep apnea. $\mathrm{SpO}_{2}$, arterial oxygen saturation. TST, total sleep time.

moderate-to-severe OSA subgroups were comparable in age, body mass index (BMI), Mallampati score, neck circumference, degree of $\mathrm{SpO}_{2}$ desaturation and optimal CPAP pressures. We initially studied all patients as a group $(\mathrm{N}=26)$. No statistically significant difference was found when comparing the AHI, the $\mathrm{SpO}_{2}$ nadir (Figure 1), the snoring index or the snoring scale on the diagnostic PSG to those on the chinstrap study (Table 2A). Similarly, in neither the mild OSA $(n=12)$ nor the moderate-tosevere OSA $(n=14)$ subgroups did the AHI or the $\mathrm{SpO}_{2}$ nadir significantly improve with chinstrap use (Tables $2 \mathrm{~B}$ and $2 \mathrm{C}$ ).

Thirteen patients had REM sleep in all 3 parts of the study, and 20 patients had supine sleep in all 3 parts of the study. Table $3 \mathrm{~A}$ presents data on TST and time spent by patients in REM sleep and while supine during each of the 3 studies. As expected given the design of the study, TST was significantly higher during the diagnostic PSG study when compared to the chinstrap and optimal CPAP studies, but was comparable between the chinstrap and optimal CPAP studies. Similarly, there was a statistically significant difference in REM sleep as a percentage of TST between the diagnostic PSG study and the chinstrap study, and between the chinstrap study and the optimal CPAP study, but not when the diagnostic PSG study and the optimal CPAP studies were compared. The percentage of TST spent in the supine position was significantly greater during the optimal CPAP titration study than during the diagnostic PSG, although comparable to the chinstrap study. We attempted to determine if chinstrap use improved SDB specifically in REM sleep (in those patients who had REM sleep in all 3 parts of the study) or in supine sleep (in those patients who had supine sleep in all 3 parts of the study), but again, could not demonstrate statistically significant improvement in the AHI with chinstrap use in either case (Table 3B). In all cases, the $\mathrm{AHI}$ and $\mathrm{SpO}_{2}$ nadir improved to a statistically significant degree at the optimal CPAP pressure (Tables $\mathbf{2}$ and $\mathbf{3}$ ).

\section{DISCUSSION}

The results of our study suggest that a chinstrap alone is an ineffective treatment modality for either OSA or snoring. The use of a chinstrap alone did not significantly improve the AHI or $\mathrm{SpO}_{2}$ nadir when compared to treatment with CPAP, the gold
Figure 1-Comparison of the effects of chinstrap and optimal CPAP pressures on sleep disordered breathing in all patients tested in the study.
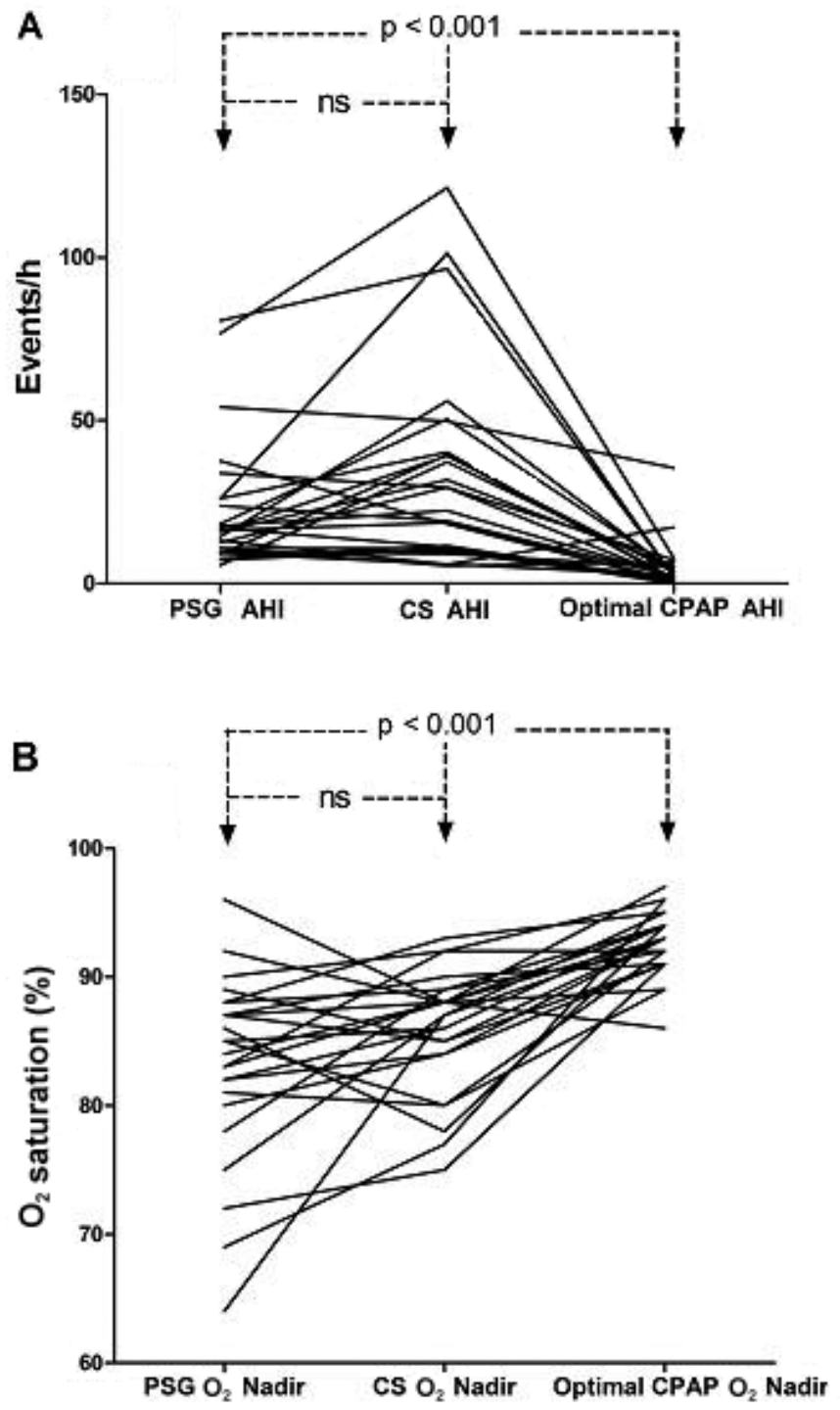

(A) Effect on apnea-hypopnea index (AHI) (B) Effect on minimum $\mathrm{SpO}_{2}$ $\left(\mathrm{O}_{2}\right.$ nadir). $\mathrm{PSG}$, diagnostic polysomnogram. CS, chinstrap study. 
Table 2-Comparison of respiratory parameters across the diagnostic PSG study, chinstrap study, and optimal CPAP study

\section{A. All patients $(\mathrm{N}=26)$}

$\mathrm{AHI}$ (events/h)

$\mathrm{SpO}_{2}$ nadir (in percentage)

Snoring Index (events/h)

Snoring Scale

\section{B. Mild OSA subgroup $(n=12)$}

$\mathrm{AHI}$ (events/h)

$\mathrm{SpO}_{2}$ nadir (in percentage)

\section{Moderate-to-severe OSA subgroup $(n=14)$}

AHI (events/h)

$\mathrm{SpO}_{2}$ nadir (in percentage)

\author{
Diagnostic PSG study \\ $16.0(9.7-26.0)$ \\ $84.0(80.5-87.5)$ \\ $253.2(147.5-353.1)$ \\ $3.0(0.8-3.0)$
}
Diagnostic PSG study
$9.6(8.1-12.2)$
$87.0(83.0-90.0)$
Diagnostic PSG study
$25.0(16.9-41.0)$
$82.0(78.0-86.0)$

\author{
Chinstrap study \\ 25.9 (10.7-42.7) \\ 87 (84.0-88.5) \\ $180(9.8-393.1)$ \\ $2.5(0.4-3.0)$
}

$$
\begin{aligned}
& \text { Optimal CPAP study } \\
& \begin{array}{l}
2.4(1.0-5.3)^{*} \\
93.0(91.0-94.0)^{*} \\
0.0(0.0-4.9)^{*} \\
0.0(0.0-0.0)^{*}
\end{array}
\end{aligned}
$$

Optimal CPAP study
$3.2(0.9-6.3)^{* *}$
$93.0(92.0-94.0)^{* *}$

Optimal CPAP study

$2.2(1.0-4.5)$ *

$92.0(91.0-94.0)$ *

All values expressed as median, interquartile range. AHI, apnea-hypopnea index; CPAP, continuous positive airway pressure; OSA, obstructive sleep apnea; PSG, polysomnogram; $\mathrm{SpO}_{2}$, arterial oxygen saturation. * Statistically significant difference between diagnostic PSG study and optimal CPAP study $(p<0.001) .{ }^{*}$ Statistically significant difference between diagnostic PSG study and optimal CPAP study $(p<0.05)$.

\section{Table 3}

$\begin{aligned} & \text { A. Amounts of sleep and percentages of REM and supine sleep in the diagnostic PSG study, chinstrap study, and on optimal CPAP pressures } \\ & \text { Diagnostic PSG study }\end{aligned}$
$\begin{array}{lccc}\text { Chinstrap study } & 136.3(119.4-157.4)^{*} & 84.6(30.4-126.9)^{*} \\ \text { TST (minutes) } & 370.0(349.9-397.5) & 8.7(42.0-100.0)^{* *} & 25.5(13.7-52.5)^{* *} \\ \text { REM sleep (as percentage of TST) } & 20.2(15.1-22.8) & 73.2(42.0-100.0)^{* * *} & 100(54.0-107.0)\end{array}$

\section{B. Comparison of AHI across the diagnostic PSG study, chinstrap study and optimal CPAP study during REM and supine sleep Diagnostic PSG study Chinstrap study \\ AHI in REM sleep only (events/h) ${ }^{a} \quad 26.7(16.8-43.7) \quad 42.2(21.3-57.7)$ \\ AHI in supine sleep only (events/h) $\quad 24.9(11.9-51.5) \quad 29.8(11.7-55.5)$

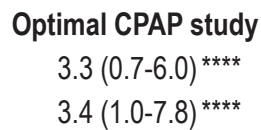 \\ Optimal CPAP study
$3.3(0.7-6.0)^{\star * * *}$
$3.4(1.0-7.8)^{\star * * *}$}

All values expressed as median, interquartile range. AHI, apnea-hypopnea index; CPAP, continuous positive airway pressure; OSA, obstructive sleep apnea; PSG, polysomnogram; REM, rapid eye movement; TST, total sleep time. *Statistically significant difference between diagnostic PSG study and chinstrap study, and between diagnostic PSG study and optimum CPAP study $(p<0.001)$. ${ }^{*}$ Statistically significant difference between diagnostic PSG study and chinstrap study $(p=0.0025)$, and between chinstrap study and optimal CPAP titration study $(p<0.01) .{ }^{* *}$ Statistically significant difference between diagnostic PSG study and optimal CPAP study $(p<0.05)$. ${ }^{* * *}$ Statistically significant difference between diagnostic PSG study and optimal CPAP study ( $\left.<<0.01\right)$. ${ }^{a}$ Includes only patients who had REM sleep in all three studies $(n=13) .{ }^{b}$ Includes only patients who had supine sleep in all three studies $(n=20)$.

standard treatment for these conditions. These parameters did not improve with chinstrap use in patients in either the mild OSA or moderate-to-severe OSA subgroups, nor did the AHI with chinstrap use improve in supine sleep or in REM sleep. The higher median AHI in REM sleep during the chinstrap study was nonsignificant and likely attributable to the significantly lower percentage of REM sleep. Also, while many patients anecdotally report that using a chinstrap improves snoring, our data showed that while this was the case with CPAP, it could not be demonstrated with chinstrap use alone.

To our knowledge, this is the first major published study that explores the questions raised by the case reported by Vorona et al. ${ }^{12}$ The mechanism by which the use of a chinstrap alone improved OSA in the patient reported by this group is not immediately apparent. Their patient was described as having a Mallampati score of 1, a normal sized tongue, and no retrognathia. They performed cephalometric analysis with and without the chinstrap in place and found no change in the posterior airway space. They also performed a flexible nasopharyngolaryngoscopy with the patient supine and found that application of the chinstrap with resultant mouth closure caused an improvement of the posterior airway space at the base of the tongue and the epiglottis, suggesting that, in their patient, the area of upper airway collapsibility was supraglottic/retroglossal rather than retropalatal. The patients in our study, on the other hand, had significant narrowing of the airway at the retropalatal level, as indicated by the high median Mallampati score. We did not perform cephalometry or nasopharyngoscopy in any of our patients in view of the lack of efficacy of the chinstrap alone in treating their OSA, and therefore do not have information regarding the degree of retroglossal narrowing in them. Several groups have made the observation that mouth opening in sleep or under sedation increases upper airway resistance and collapsibility based on critical pressure 
and resistance measurements. ${ }^{15,16}$ Furthermore, Lee et al. ${ }^{17}$ used lateral cephalometry and fiberoptic nasopharyngoscopy and found that open-mouth breathing during sleep caused reduction of the retropalatal and retroglossal areas, lengthening of the pharynx and shortening of the mandible-hyoid distance in the upper airway, thereby worsening OSA. These findings were supported by an interesting study published by Kim et al., who employed three-dimensional multi-detector computed tomography and found that open-mouth breathing decreased the minimal cross-sectional area of the retropalatal and retroglossal regions. ${ }^{18}$ While our study suggests that patients with upper airway narrowing at the retropalatal level would not benefit from a chinstrap alone for treatment of OSA, the intriguing question remains as to whether mouth closure by a chinstrap alone would improve SDB in those patients whose area of anatomical collapsibility is predominantly in the posterior airway (supraglottic/retroglossal). Future studies might evaluate this in patients with predominantly posterior airway narrowing on nasopharyngoscopy and lateral cephalometry.

Recently, Tsuda et al. studied mandibular position in sleep in patients with OSA using jaw position sensors, and found that mouth opening progressively increased as patients progressed from stage N1 to REM sleep. ${ }^{19}$ Although they could not establish a causal relationship between the degree of mouth opening and the severity of OSA, it would be reasonable to hypothesize that, since mouth breathing has been implicated in increased upper airway collapsibility, mouth closure with a chinstrap may improve the AHI to a greater degree in REM sleep. However, our study did not support this hypothesis, as there was no improvement in the AHI in REM sleep with chinstrap use in our patients. Similarly, Miyamoto et al. found that the degree of mouth opening in sleep was greater in patients with OSA compared to controls, and that in the supine position, this was dependent on sleep stage, increasing progressively during apneic episodes and decreasing at the termination of episodes in NREM sleep but not in REM sleep. ${ }^{20}$ This raises the question of whether there would be improvement in the AHI during supine sleep with a chinstrap. Again, however, our data failed to demonstrate such an improvement.

A few key differences between the design of our study and the case reported by Vorona ${ }^{12}$ deserve mention. The patient reported by Vorona had his chinstrap PSG performed three months after his initial PSG. He had used CPAP for approximately a month, but had stopped using it for about two months. It is therefore unlikely that any residual effects of CPAP use on upper airway anatomy or edema would have persisted by the time of the chinstrap PSG, as the authors themselves have pointed out. In contrast, our patients were CPAP-naïve when they underwent their chinstrap studies. However, for the reasons mentioned above, we do not believe this invalidates our results. In any event, if the residual effect of CPAP use is the reason for the improvement of the patient's AHI in the second PSG by Vorona et al., then it would argue against a chinstrap alone being an effective treatment for OSA.

While our study may have used a different brand and design of chinstrap than the one used by Vorona's group (which was not specified in their published report), we do not believe it is likely that this factor would account for the lack of efficacy of chinstrap use in treating OSA in our patients. Similarly, while we did not study the commercially available and much more expensive chinstraps being marketed as standalone "snoring solutions" and treatments for sleep apnea, it seems unlikely that these devices, which do not appear to be fundamentally different from the typical chinstraps used to keep the mouth closed during CPAP use and which we used in our study, would produce different results unless there were a mechanism by which they could thrust the jaw forward, which does not appear to be the case based on a review of their brochures and websites.

There are some inherent criticisms of our study. It is conceivable that the differences in TST during the diagnostic, chinstrap, and optimal CPAP studies may have influenced the respective AHIs. Due to insurance-mandated directives, we were unable to perform full-night chinstrap PSGs, except in one patient, and therefore were forced to limit the chinstrap portion of the diagnostic split-night study to approximately two hours. Unsurprisingly, therefore, the TST was significantly higher during the diagnostic PSG studies compared to the chinstrap and optimal CPAP studies, although it was comparable between the chinstrap and optimal CPAP titration studies. Nevertheless, one consequence of this situation is the low percentages and lack of REM sleep in many patients during the chinstrap study. While shorter recording times might have resulted in higher AHIs during the chinstrap study, the converse argument would be that the lower percentage of REM sleep in this part of the study, as well as the fact that the chinstrap study was performed in the first part of the night when OSA is generally less severe than in the latter part, would work in favor of a lower chinstrap AHI. Supine sleep, which would be expected to worsen AHIs, was not present in significantly greater amounts in the chinstrap study compared to the diagnostic study, although it occurred in greater amounts during the optimal CPAP study. Ultimately, however, it is difficult to analyze how much of a role these factors may have played in the AHIs reported in the three parts of the study, and this should be borne in mind while interpreting the results. It bears remembering that Vorona et al. did not feel that the improvement in their patient's AHI with chinstrap use was related to sleep stage or position, and they did not report separate AHIs based on stage or position. ${ }^{12}$

With regards to the findings related to snoring, we do acknowledge that the snoring index lacks validation as a standardized measure of the severity of snoring, and may not be reflective of its degree of obnoxiousness in the real world. For this reason, we also compared changes in the snoring scale, which was based on the technician's subjective impression about snoring intensity, similar to those used in other studies. ${ }^{21}$ The non-standardized nature of the former measure and the subjective nature of the latter notwithstanding, we still feel that our findings are of interest, since from a practical point of view, snoring is a subjective symptom most disruptive and burdensome to a patient's bed partner. From a patient perspective, the elimination or reduction of snoring intensity is the ultimate goal, and we feel that the report of a technician observing the patient during a sleep study would be a reasonable means of comparison. Also, the lack of a universally accepted method to evaluate snoring during sleep studies means that any report of snoring is, by its very nature, non-standardized..$^{22}$ In many cases, snoring is evaluated based on questionnaires completed by a patient's bed partner, and a future study might consider 
evaluating a chinstrap for snoring over several nights through such a questionnaire to evaluate the home bedroom setup and to account for night-to-night variability and the sensitivity of the bed partner to the patient's snoring. ${ }^{23}$

In conclusion, our study suggests that the use of a chinstrap alone does not improve the AHI or oximetry results in patients with OSA, even with mild disease, and is an ineffective treatment for this condition. It also did not improve SDB during REM or supine sleep, suggesting that it would not be a viable option in patients with purely REM-related or positional OSA. In addition, it is also an ineffective snoring solution. It is important for sleep physicians and primary care physicians to be aware of these findings, as patients who read about this treatment modality on the internet often enquire about its effectiveness.

\section{REFERENCES}

1. Peppard PE, Young T, Barnet JH, Palta M, Hagen EW, Hla KM. Increased prevalence of sleep-disordered breathing in adults. Am $\mathrm{J}$ Epidemiol 2013;177:1006-14.

2. Sanna A. Obstructive sleep apnoea, motor vehicle accidents, and work performance. Chron Respir Dis 2013;10:29-33.

3. Bista SR, Barkoukis TJ. Medical disorders impacted by obstructive sleep apnea. Dent Clin North Am 2012;56:373-86.

4. Mihălțan F, Deleanu O, Munteanu I, Ulmeanu R, Nemeş R, Oros M.[Adherence to CPAP- "eternal" issue]. [Article in Romanian] Pneumologia 2012;61:108-12.

5. Reeves-Hoche MK, Meck R, Zwillich CW. Nasal CPAP: an objective evaluation of patient compliance. Am J Respir Crit Care Med 1994;149:149-54.

6. Waldhorn RE, Herrick TW, Nguyen MC, O'Donnell AE, Sodero J, Potolicchio SJ. Long-term compliance with nasal continuous positive airway pressure therapy of obstructive sleep apnea. Chest 1996;97:33-8.

7. $\mathrm{Ng} \mathrm{A}$, Gotsopoulos H, Darendeliler AM, Cistulli PA. Oral appliance therapy for obstructive sleep apnea. Treat Respir Med 2005;4:409-22.

8. Farid-Moayer M, Siegel LC, Black J. A feasibility evaluation of oral pressure therapy for the treatment of obstructive sleep apnea. Ther Adv Respir Dis 2013;7:3-12

9. Kryger MH, Berry RB, Massie CA. Long-term use of a nasal expiratory positive airway pressure (EPAP) device as a treatment for obstructive sleep apnea (OSA). J Clin Sleep Med 2011;7:449-53B.

10. Carvalho B, Hsia J, Capasso R. Surgical therapy of obstructive sleep apnea: a review. Neurotherapeutics 2012;9:710-6.

11. Li KK. Sleep apnea surgery: putting it all together. Oral Maxillofac Surg Clin North Am 2009;21:421-3.

12. Vorona RD, Ware JC, Sinacori JT, Ford ML 3rd, Cross JP. Treatment of severe obstructive sleep apnea syndrome with a chinstrap. J Clin Sleep Med 2007;3:729-30.

13. Iber C, Ancoli-Israel S, Chesson AL, Quan SF. The AASM manual for the scoring of sleep and associated events: rules, terminology, and technical specifications. Westchester, IL: American Academy of Sleep Medicine, 2007.
14. Randles RH, Wolfe DA. Introduction to the theory of nonparametric statistics. New York: Wiley, 1979.

15. Meurice JC, Marc I, Carrier G, Series F. Effects of mouth opening on upper airway collapsibility in normal sleeping subjects. Am J Respir Crit Care Med 1996;153:255-9.

16. Ayuse $T$, Inazawa $T$, Kurata $S$, et al. Mouth-opening increases upper-airway collapsibility without changing resistance during midazolam sedation. J Dent Res 2004;83:718-22.

17. Lee SH, Choi JH, Shin C, Lee HM, Kwon SY, Lee SH. How does open-mouth breathing influence upper airway anatomy? Laryngoscope 2007;117:1102-6.

18. Kim EJ, Choi JH, Kim KW, et al. The impacts of open-mouth breathing on upper airway space in obstructive sleep apnea: 3-D MDCT analysis. Eur Arch Otorhinolaryngol 2011;268:533-9.

19. Tsuda H, Lowe AA, Chen H, Fleetham JA, Ayas NT, Almeida FR. The relationship between mouth opening and sleep stage-related sleep disordered breathing. J Clin Sleep Med 2011;7:181-6

20. Miyamoto K, Ozbek MM, Lowe AA, Sjöholm TT, Love LL, Fleetham JA, Ryan CF. Mandibular posture during sleep in patients with obstructive sleep apnoea. Arch Oral Biol 1997:44:657-64.

21. Hoffstein V, Mateika S, Anderson D. Snoring: is it in the ear of the beholder? Sleep 1994;17:522-6.

22. Hoffstein V, Mateika S, Nash S. Comparing perceptions and measurements of snoring. Sleep 1996;19:783-9.

23. Dreher A, Rader T, Patscheider M, et al. The annoyance of snoring. Eur Arch Otorhinolaryngol 2009;266:293-6.

\section{ACKNOWLEDGMENTS}

The authors express their gratitude to the following registered polysomnography technicians for their invaluable assistance with performing this study; Stacey Adams, Eddy Canela, Ana Ferreira, Giulia King-Tapia, Rhonda McLemore, Carlos Navarro, Patrick Protacio, Richard Pugh, Mitchell Rubinstein, Bindi Shah, Roderick Tero, and Sherise Vargas.

\section{SUBMISSION \& CORRESPONDENCE INFORMATION}

Submitted for publication October, 2013

Submitted in final revised form March, 2014

Accepted for publication March, 2014

Address correspondence to: Sushanth Bhat, M.D., 1NJ Neuroscience Institute at JFK Medical Center/Seton Hall University, 65 James Street, Edison NJ 08818; Tel: (732) 321-7010; Fax: (732) 205-1477; E-mail: sbhat2012@yahoo.com

\section{DISCLOSURE STATEMENT}

This was not an industry supported study. The work was performed at NJ Neuroscience Institute at JFK Medical Center/Seton Hall University, Edison NJ, The authors have indicated no financial conflicts of interest. 